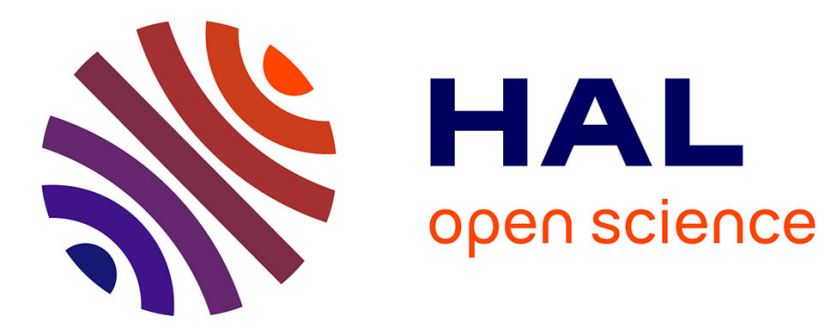

\title{
Asymptotic Karlin-Rubin's Theorem with Application to Signal Detection in a Subspace Cone
}

\author{
Sabrina Bourmani, François-Xavier Socheleau, Dominique Pastor
}

\section{To cite this version:}

Sabrina Bourmani, François-Xavier Socheleau, Dominique Pastor. Asymptotic Karlin-Rubin's Theorem with Application to Signal Detection in a Subspace Cone. EUSIPCO 2019: 27th European Signal

Processing Conference, Sep 2019, Coruna, Spain. 10.23919/EUSIPCO.2019.8902793 . hal-02272526

\section{HAL Id: hal-02272526 \\ https://hal.science/hal-02272526}

Submitted on 27 Aug 2019

HAL is a multi-disciplinary open access archive for the deposit and dissemination of scientific research documents, whether they are published or not. The documents may come from teaching and research institutions in France or abroad, or from public or private research centers.
L'archive ouverte pluridisciplinaire HAL, est destinée au dépôt et à la diffusion de documents scientifiques de niveau recherche, publiés ou non, émanant des établissements d'enseignement et de recherche français ou étrangers, des laboratoires publics ou privés. 


\title{
Asymptotic Karlin-Rubin's Theorem with Application to Signal Detection in a Subspace Cone
}

\author{
Sabrina Bourmani, Francois-Xavier Socheleau, Dominique Pastor \\ IMT Atlantique, Lab-STICC, Bretagne Loire University \\ Technopole Brest-Iroise CS83818, Brest 29238, France \\ \{sabrina.bourmani, fx.socheleau, d.pastor\}@imt-atlantique.fr
}

\begin{abstract}
We first propose an asymptotic formulation of Karlin-Rubin's theorem that relies on the weak convergence of a sequence of random vectors to design Asymptotically Uniformly Most Powerful (AUMP) tests dedicated to composite hypotheses. This general property of optimality is then applied to the problem of testing whether the energy of a signal projected onto a known subspace exceeds a specified proportion of its total energy. The signal is assumed unknown deterministic and it is observed in independent and additive white Gaussian noise. Such a problem can arise when the signal to be detected obeys the linear subspace model and when it is corrupted by unknown interference. It can also be relevant in machine learning applications where one wants to check whether an assumed linear model fits the analyzed data. For this problem, where it is shown that no Uniformly Most Powerful (UMP) and no UMP invariant tests exist, an AUMP invariant test is derived.
\end{abstract}

\section{INTRODUCTION}

The most desirable way to solve a hypothesis-testing problem is to find a statistical test that is optimal according to a certain criterion. Uniformly Most Powerful (UMP) tests are often looked for as they provide the greatest power among the class of tests with the same level. Neyman-Pearson's lemma provides UMP tests for simple hypotheses and Karlin Rubin's theorem extends this lemma to composite hypotheses. The latter states that the comparison of a scalar statistic to a threshold leads to a UMP test if this statistic has monotone nondecreasing likelihood ratio [1]-[3]. However, in many practical situations, no UMP tests exist due to the lack of knowledge on the parameters involved in the problem at hand. In such a situation, attention is often restricted to a specific class of tests (e.g., invariant tests) and/or tests that show asymptotic optimality.

In this paper, we first propose an asymptotic formulation of the Neyman-Pearson and Karlin-Rubin theorems that relies on the weak convergence of a sequence of random vectors and the concept of Asymptotically Uniformly Most Powerful (AUMP) test dedicated to composite hypotheses, such a concept being inspired by [2, Def. 13.3.2]. These general results are then applied to the problem of testing whether a signal, observed in Gaussian noise, belongs to a subspace cone.

Linear subspaces are commonly used to model structured signals/data [3]-[6]. The detection of such signals observed in additive noise is classically performed thanks to matched subspace detectors [7]. However, in many applications there may be uncertainty about the signal (or subspace) of interest
[8]-[10] and interference may also be present in addition to noise [7], [11], [12]. In some cases, the interference subspace is either known or learned from training data [7] and in others [11], [12], the interference is so poorly known that no parametric model can be used. Signal uncertainties due to model mismatch and/or to the presence of poorly known interference can be modeled by a subspace cone [8], [9], [11], [12]. In the present work, the cone is defined as a boundary in the measurement space delimited by the set of all signals which possess a specified fraction $\tau$ of their total energy in a known linear subspace. On the basis of noisy observations, we hereafter address the problem of testing whether a signal belongs to the cone $\left(\mathcal{H}_{1}\right)$ or not $\left(\mathcal{H}_{0}\right)$. The signal is assumed to be unknown deterministic and it is observed in independent and additive white Gaussian noise. The invariances of the problem are described and it is shown that no Uniformly Most Powerful Invariant (UMPI) test exists. A test relying on a maximal invariant statistics is derived and found to be asymptotically UMPI thanks to the proposed Karlin-Rubin theorem.

The concept of AUMP tests is introduced in Sec. II along with additional definitions and notation. The asymptotic Karlin-Rubin theorem is stated in Sec. III-B and applied to the subspace cone detection problem IV-A. Numerical examples are provided in Sec. V, followed by conclusions in Sec. VI.

\section{DEFINITIONS AND NOTATION}

Definition 1. A test is an application $\mathcal{T}: \Gamma \rightarrow\{0,1\}$ such that

$$
\mathcal{T}(\boldsymbol{y})=\left\{\begin{array}{l}
1 \text { if } \boldsymbol{y} \in \mathcal{R}^{\mathcal{T}} \\
0 \text { if } \boldsymbol{y} \in \overline{\mathcal{R}^{\mathcal{T}}}
\end{array}\right.
$$

with $\mathcal{R}^{\mathcal{T}} \cup \overline{\mathcal{R}^{\mathcal{T}}}=\Gamma$ and $\mathcal{R}^{\mathcal{T}} \cap \overline{\mathcal{R}^{\mathcal{T}}}=\varnothing . \mathcal{R}^{\mathcal{T}}$ is called the critical region of the test $\mathcal{T}$.

By definition 1, we hereafter focus on non-randomized tests only.

Definition 2. Let $\boldsymbol{Y}$ be a random vector. $A$ set $\mathcal{B}$ is called a $\mathbb{P}$-continuity set if $\mathbb{P}[\boldsymbol{Y} \in \partial \mathcal{B}]=0$, where $\partial \mathcal{B}$ is the border of $\mathcal{B}$ [13, Ch. 1].

Definition 3. Let $\left(\boldsymbol{Y}_{n}\right)_{n \in \mathbb{N}^{*}}$ be a sequence of random vectors of probability density function $f_{\boldsymbol{Y}_{n}}^{\theta}(\boldsymbol{y})$ with $\theta \in \Theta$. We consider the following detection problem

$$
\left\{\begin{array}{l}
\mathcal{H}_{0}: \theta \in \Theta_{0} \\
\mathcal{H}_{1}: \theta \in \Theta_{1}, \quad \Theta_{0} \cup \Theta_{1}=\Theta, \Theta_{0} \cap \Theta_{1}=\varnothing
\end{array}\right.
$$


A test $\mathcal{T}$ is called AUMP (Asymptotically Uniformly Most Powerful) of asymptotic size $\alpha$ if

$$
\lim _{n} \sup _{\theta \in \Theta_{0}} \mathbb{E}_{\theta}\left[\mathcal{T}\left(\boldsymbol{Y}_{n}\right)\right]=\alpha,
$$

and if for any other test $\mathcal{T}^{\prime}$ satisfying

$$
\lim _{n} \sup _{\theta \in \Theta_{0}} \mathbb{E}_{\theta}\left[\mathcal{T}^{\prime}\left(\boldsymbol{Y}_{n}\right)\right] \leq \alpha,
$$

we have, $\lim _{n} \sup _{\theta \in \Theta_{1}}\left(\mathbb{E}_{\theta}\left[\mathcal{T}^{\prime}\left(\boldsymbol{Y}_{n}\right)\right]-\mathbb{E}_{\theta}\left[\mathcal{T}\left(\boldsymbol{Y}_{n}\right)\right]\right) \leq 0$.

The asymptotic optimality considered here is inspired by [2, Def. 13.3.2]. In contrast to this reference, our definition concerns composite hypotheses and involves limits in $n$ instead of limsup's.

Notation: We note $\xi_{\boldsymbol{Y}}$ the family of tests whose critical regions are $\mathbb{P}$-continuity sets of the random vector $\boldsymbol{Y}$, i.e., $\xi_{\boldsymbol{Y}} \triangleq\left\{\mathcal{T}: \mathbb{P}\left[\boldsymbol{Y} \in \partial \mathcal{R}^{\mathcal{T}}\right]=0\right\}$. The convergence in distribution of a sequence of random variables $\left(Y_{n}\right)_{n \in \mathbb{N}^{*}}$ to a random variable $Y$ is denoted as $Y_{n} \stackrel{\mathcal{L}}{\rightarrow} Y$. The convergence in probability is denoted as $Y_{n} \stackrel{\mathbb{P}}{\rightarrow} Y$. The superscripts ${ }^{T}$ denotes matrix transposition and $\|\cdot\|$ designates the standard Euclidean norm. The distribution of a non-central chi-squared distribution with $\nu$ degrees of freedom and noncentrality parameter $\ell$ is denoted $\chi_{\nu}^{2}(\ell) . \mathcal{F}\left(\nu_{1}, \nu_{2}, \ell_{1}, \ell_{2}, \cdot\right)$ denotes the probability density function (pdf) of a doubly non-central $\mathcal{F}$-distribution that corresponds to the ratio of two independent noncentral chi-squared variables, normalized by their degree of freedom $\nu_{1}$ and $\nu_{2}$, respectively, and of non centrality parameters $\ell_{1}$ and $\ell_{2}$. The corresponding cumulative distribution is denoted as $F\left(\nu_{1}, \nu_{2}, \ell_{1}, \ell_{2}, \cdot\right)$.

III. AN ASYMPTOTIC FORMULATION OF KARLIN-RUBIN'S THEOREM

\section{A. Preliminary material}

Proposition 1 (Asymptotic Neyman-Pearson's lemma). Let $\left(\boldsymbol{Y}_{n}\right)_{n \in \mathbb{N}^{*}}$ be a sequence of random vectors, where every $\boldsymbol{Y}_{n}$ has a pdf $f_{\boldsymbol{Y}_{n}}^{\theta}(\boldsymbol{y}), \theta \in \Theta \subset \mathbb{R}$. Let $\boldsymbol{Y}$ be an absolutely continuous random vector with pdf $f_{\boldsymbol{Y}}^{\theta}(\boldsymbol{y}), \theta \in \Theta$. Suppose the existence of $\varphi \in \xi_{\boldsymbol{Y}}$ such that

$$
\varphi(\boldsymbol{y})= \begin{cases}1 & \text { if } f_{\boldsymbol{Y}}^{\theta_{1}}(\boldsymbol{y}) \geq \eta f_{\boldsymbol{Y}}^{\theta_{0}}(\boldsymbol{y}) \\ 0 & \text { otherwise, }\end{cases}
$$

where $\eta \geq 0$ and $\mathbb{E}_{\theta_{0}}\{\varphi(\boldsymbol{Y})\}=\alpha$.

If $\boldsymbol{Y}_{n} \stackrel{\mathcal{L}}{\rightarrow} \boldsymbol{Y}$, then $\varphi$ is AUMP of asymptotic level $\alpha$ for testing

$$
\left\{\begin{array}{l}
\mathcal{H}_{0}: \theta=\theta_{0} \\
\mathcal{H}_{1}: \theta=\theta_{1}
\end{array}\right.
$$

among all tests belonging to $\xi_{\boldsymbol{Y}}$ and $\varphi$ is asymptotically unbiased, i.e. $\mathbb{E}_{\theta_{1}}[\varphi(\boldsymbol{Y})] \geq \mathbb{E}_{\theta_{0}}[\varphi(\boldsymbol{Y})]=\alpha$.

Proof. See Appendix A.

\section{B. Asymptotic Karlin-Rubin's theorem}

Proposition 2. Let $\boldsymbol{Y}: \Omega \rightarrow \mathbb{R}^{\mathbb{N}}$ be an absolutely continuous random vector of pdf $f_{\boldsymbol{Y}}^{\theta}(\boldsymbol{y})$, where $\theta \in \Theta \subset \mathbb{R}$, and let $\Theta_{1}$ and $\Theta_{0}$ be two subsets of $\Theta$ such that: $\Theta=\Theta_{0} \cup \Theta_{1}$ and $\Theta_{0} \cap \Theta_{1}=\varnothing$. We assume that $f_{\boldsymbol{Y}}^{\theta_{0}}(\boldsymbol{y}) \neq 0$ for all $\boldsymbol{y} \in \mathbb{R}^{N}$.

Let:

$$
\Lambda_{\theta_{0}, \theta_{1}}(\boldsymbol{y})=\frac{f_{\boldsymbol{Y}}^{\theta_{1}}(\boldsymbol{y})}{f_{\boldsymbol{Y}}^{\theta_{0}}(\boldsymbol{y})}
$$

with $\theta_{0} \in \Theta_{0}$ and $\theta_{1} \in \Theta_{1}$ be the likelihood ratio such that $\Lambda_{\theta_{0}, \theta_{1}}=h_{\theta_{0}, \theta_{1}} \circ V$, with $V: \mathbb{R}^{N} \mapsto \mathbb{R}$.

Let $\left(\boldsymbol{Y}_{n}\right)_{n \in \mathbb{N}^{*}}$ be a sequence of random vectors. If, for any $\theta_{0}<\theta_{1}, h_{\theta_{0}, \theta_{1}}$ is strictly increasing in $V$ and $\boldsymbol{Y}_{n} \stackrel{\mathcal{L}}{\rightarrow} \boldsymbol{Y}$, then the test

$$
\varphi^{\prime}(\boldsymbol{y})= \begin{cases}1 & \text { if } V(\boldsymbol{y}) \geq \lambda \\ 0 & \text { otherwise }\end{cases}
$$

with

$$
\mathbb{E}_{\theta_{0}}\left[\varphi^{\prime}(\boldsymbol{Y})\right]=\mathbb{P}_{\theta_{0}}[V(\boldsymbol{Y}) \geq \lambda]=\alpha,
$$

is AUMP among all tests belonging to $\xi_{\boldsymbol{Y}}$ for testing

$$
\left\{\begin{array}{l}
\mathcal{H}_{0}^{\prime}: \theta \leq \theta_{0} \\
\mathcal{H}_{1}^{\prime}: \theta>\theta_{0}
\end{array}\right.
$$

Proof. See Appendix B.

\section{Application : testing the presence of a signal in a Subspace Cone}

\section{A. Problem statement}

Let $\boldsymbol{z} \in \mathbb{R}^{N}$ denote an unknown deterministic signal observed in white Gaussian noise $\boldsymbol{w} \sim \mathcal{N}\left(0, \sigma^{2} \mathbf{I}_{N}\right)$ with unknown variance $\sigma^{2}$. The observation vector is modeled as

$$
\boldsymbol{y}=\boldsymbol{z}+\boldsymbol{w} .
$$

The signal-to-noise ratio (SNR), defined as $\gamma=\frac{\|z\|^{2}}{N \sigma^{2}}$, is assumed to be known for now. This assumption will be relaxed in Sec. IV-D. Let $\mathbf{H}$ denote an $N \times n$ matrix that spans a rank- $n$ subspace $\langle\mathbf{H}\rangle$ with $n \leqslant N$ and $\mathbf{P}_{\mathbf{H}}$ its corresponding projection matrix, i.e., $\mathbf{P}_{\mathbf{H}}=\mathbf{H}\left(\mathbf{H}^{T} \mathbf{H}\right)^{-1} \mathbf{H}^{T}$.

Given a tolerance $0 \leq \tau \leq 1$, let $\mathcal{C}_{\mathbf{H}}$ denote the subspace cone defined as $\mathcal{C}_{\mathbf{H}}=\left\{\boldsymbol{x} \in \mathbb{R}^{N}: \rho=\frac{\left\|\mathbf{P}_{\mathbf{H}} \boldsymbol{x}\right\|^{2}}{\|\boldsymbol{x}\|^{2}}>\tau\right\}$. We address the problem of testing whether $\boldsymbol{z}$ belongs to the cone $\mathcal{C}_{\mathbf{H}}$ or not, when we are given $\boldsymbol{y}$. The hypothesis testing problem is therefore

$$
\left\{\begin{array}{l}
\mathcal{H}_{0}: z \notin \mathcal{C}_{\mathbf{H}} \\
\mathcal{H}_{1}: z \in \mathcal{C}_{\mathbf{H}}
\end{array}\right.
$$

Problem (11) amounts to testing whether a sufficient proportion of the signal energy lies in the subspace $\langle\mathbf{H}\rangle$. Such a problem formulation can be relevant when the signal to be detected obeys the linear subspace model and when it is corrupted by some poorly known interference $\boldsymbol{\zeta}$, i.e., $z=$ $\mu \mathbf{H} \psi+\zeta$, where $\mu$ is a random variable valued in $\{0,1\}$ modeling the possible presence or absence of the signal, and where $\psi \in \mathbb{R}^{n}$ is the unknown coordinates of this signal in the subspace $\langle\mathbf{H}\rangle$. For instance, in some applications [12], [14], interference can be so heterogeneous that it cannot be 
represented by a single parametric model and that the detector cannot learn, thanks to a training dataset, the common features shared by these interfering signals. In such cases, $\zeta$ cannot be assumed to be orthogonal to the signal of interest or to obey a known subspace model [7]. Given the poor knowledge available on the interference, $\boldsymbol{\zeta}$ can only be loosely defined as a signal having "not much in common" with the signal of interest. More formally, $\zeta$ may be defined as a vector whose energy lies mostly outside the subspace in which the signal of interest resides, i.e., $\left\|\mathbf{P}_{\mathbf{H}} \boldsymbol{\zeta}\right\|^{2} \leq \tau\|\boldsymbol{\zeta}\|^{2}$. Assuming further that $\left\|\mathbf{H} \psi+\mathbf{P}_{\mathbf{H}} \boldsymbol{\zeta}\right\|^{2}>\tau\|\mathbf{H} \boldsymbol{\psi}+\boldsymbol{\zeta}\|^{2},(11)$ is then equivalent to the problem of testing whether $\mu=0$ or $\mu=1$. A similar model is used and applied to real data in [12], [14], which attests of its practical relevance. Note that as opposed to other subspace cone detectors such as [8], [9], the null hypothesis in (11) is not restricted to the observation of Gaussian noise only. Problem (11) can also be relevant in machine learninglike applications where one wants to check on a given dataset whether the assumed linear model matches the analyzed data with a sufficient accuracy $\tau$.

\section{B. Uniformly Most Powerful Invariant Test}

Since $z$ is not known perfectly, no UMP test exists. As Problem (11) is invariant, our attention is restricted to tests invariant to sets of transformations for which the problem is itself invariant. This invariance can be formalized through the group of transforms in $\mathbb{R}^{N}$

$$
\mathcal{G}=\left\{g: g(\boldsymbol{x})=\kappa\left(\mathbf{U}_{\mathbf{H}} \mathbf{Q} \mathbf{U}_{\mathbf{H}}^{T}+\mathbf{U}_{\mathbf{H}^{\perp}} \mathbf{R} \mathbf{U}_{\mathbf{H}^{\perp}}^{\mathbf{T}}\right) \boldsymbol{x}\right\},
$$

where $\kappa \in \mathbb{R}, \mathbf{Q}$ and $\mathbf{R}$ are $n \times n$ and $(N-n) \times(N-$ $n$ ) orthogonal matrices, respectively. $\mathbf{U}_{\mathbf{H}}$ is defined such that $\mathbf{P}_{\mathbf{H}}=\mathbf{U}_{\mathbf{H}} \mathbf{U}_{\mathbf{H}}^{T}$ and $\mathbf{U}_{\mathbf{H}^{\perp}}$ such that $\mathbf{P}_{\mathbf{H}}=\mathbf{I}_{N}-\mathbf{U}_{\mathbf{H}^{\perp}} \mathbf{U}_{\mathbf{H}^{\perp}}^{\mathbf{T}}$.

Given the invariance of our problem, it is desirable to find a test $\mathcal{T}$ such that $\mathcal{T}(g(\boldsymbol{x}))=\mathcal{T}(\boldsymbol{x})$ for all $\boldsymbol{x} \in \mathbb{R}^{N}$ and all $g \in \mathcal{G}$. It can easily be checked that

$$
\boldsymbol{x} \in \mathbb{R}^{N} \mapsto M(\boldsymbol{x})=\frac{N-n}{n} \times \frac{\left\|\mathbf{P}_{\mathbf{H}} \boldsymbol{x}\right\|^{2}}{\left\|\left(\mathbf{I}_{N}-\mathbf{P}_{\mathbf{H}}\right) \boldsymbol{x}\right\|^{2}} \in[0, \infty)
$$

is a maximal invariant of $\mathcal{G}$, that is $M(\boldsymbol{x})=M(g(\boldsymbol{x}))$ for all $g \in \mathcal{G}$ and $M\left(\boldsymbol{x}_{1}\right)=M\left(\boldsymbol{x}_{2}\right) \Rightarrow \boldsymbol{x}_{2}=g\left(\boldsymbol{x}_{1}\right)$ for some $g$ $\in \mathcal{G}$. Therefore, it follows from [2, Theorem 6.2.1] that any $\mathcal{G}$-invariant test is a function of this maximal invariant.

If it exists, the UMPI test statistic can be obtained by computing the likelihood ratio of the maximal invariant $M(\boldsymbol{y})$. Since $M(\boldsymbol{y})$ can be expressed as the ratio of two (scaled) noncentral chi-square random variables, it is distributed according to a doubly non-central $\mathcal{F}$-distribution $[15, \mathrm{Ch}$. 30] so that the likelihood ratio is expressed as

$$
L_{M}(\boldsymbol{y})=\frac{\mathcal{F}\left(n, N-n, N \gamma \rho_{1}, N \gamma\left(1-\rho_{1}\right), M(\boldsymbol{y})\right)}{\mathcal{F}\left(n, N-n, N \gamma \rho_{0}, N \gamma\left(1-\rho_{0}\right), M(\boldsymbol{y})\right)}
$$

where $\rho_{0} \leq \tau$ (resp. $\rho_{1}>\tau$ ) denotes the actual percentage of the energy of $\boldsymbol{z}$ in the subspace $\langle\mathbf{H}\rangle$ under hypothesis $\mathcal{H}_{0}$ (resp. $\mathcal{H}_{1}$ ). Since the hypotheses of Problem (11) are composite, $\rho_{0}$ and $\rho_{1}$ are not provided in the problem statement so that the UMPI test cannot be implemented using $L_{M}(\boldsymbol{y})$. For composite hypothesis testing, the UMPI test can sometimes be derived by invoking Karlin Rubin's theorem. Unfortunately, $L_{M}(\boldsymbol{y})$ is not a nondecreasing function of $M(\boldsymbol{y})$ for every pair $\left(\rho_{1}>\rho_{0}, \rho_{0}\right)$ and any set of parameters $(n, N, \gamma)$. Therefore, the UMPI test does not always exist. However, for large $N$, we next show that an asymptotically UMPI test always exists.

\section{Asymptotically Uniformly Most Powerful Invariant Test}

Given $\alpha \in(0,1)$, let the test $\mathcal{T}_{\alpha}$ be defined, for any $\boldsymbol{x} \in \mathbb{R}^{N}$, as

$$
\mathcal{T}_{\alpha}(\boldsymbol{x})=\left\{\begin{array}{l}
1 \text { if } M(\boldsymbol{x}) \geq \lambda \\
0 \text { otherwise }
\end{array}\right.
$$

with $\lambda$ such that

$$
F(n, N-n, N \gamma \tau, N \gamma(1-\tau), \lambda)=1-\alpha .
$$

The power function of this test satisfies $\mathbb{P}\left[\mathcal{T}_{\alpha}(\boldsymbol{z}+\boldsymbol{w})=\right.$ $1]=1-F(n, N-n, N \gamma \rho, N \gamma(1-\rho), \lambda)$ where $\rho=$ $\left\|\mathbf{P}_{\mathbf{H}} \boldsymbol{z}\right\|^{2} /\|\boldsymbol{z}\|^{2}$.

Proposition 3. $\mathcal{T}_{\alpha}$ is AUMP with size $\alpha$ among $\mathcal{G}$-invariant tests for Problem (11).

Proof. $M(\boldsymbol{y})=(N-n) Z_{1} /\left(n Z_{2}\right)$, where $Z_{1} \sim \chi_{n}^{2}(N \gamma \rho)$ and $Z_{2} \sim \chi_{(N-n)}^{2}(N \gamma(1-\rho))$. For $n$ fixed and as $N \rightarrow \infty$, by the law of large numbers we have $: Z_{2} /(N-n) \stackrel{\mathbb{P}}{\rightarrow} 1$. Applying Slutsky's theorem, for $N \rightarrow \infty$, we get $M(\boldsymbol{y}) \stackrel{\mathcal{L}}{\rightarrow}$ $Z_{1} / n$. Since the non-central chi-squared distribution is known to have a monotone increasing likelihood ratio [16, pp. 469], Prop. 2 applies with $\Theta_{0}=[0, \tau]$ and $\Theta_{1}=(\tau, 1]$. Thence the result.

Interestingly, the maximal invariant statistic $M(\boldsymbol{y})$ is the same as the one used in other detection problems such as [7, Ch. 4.12] and [11, App. A]. However, note that the test itself is different since the power function as well as the optimality properties are problem-dependent.

\section{Unknown SNR}

Knowledge of the SNR $\gamma$ is required in (16) to compute the detection threshold $\lambda$ so as to satisfy the constraint on the size $\alpha$. In practice, $\gamma$ is rarely known perfectly so that $\mathcal{T}_{\alpha}$ cannot be strictly applied. However, $\mathcal{T}_{\alpha}$ can provide guidelines to design relevant ad-hoc tests when no optimal test exists. For instance, a robust test $\mathcal{T}_{\alpha}^{\prime}$ can be used where $M(\boldsymbol{y})$ is compared to a new threshold $\lambda^{\prime}$ such that

$$
\sup _{\gamma \in \mathcal{S}} F\left(n, N-n, N \gamma \tau, N \gamma(1-\tau), \lambda^{\prime}\right)=1-\alpha,
$$

where, (i) $\mathcal{S}=\mathbb{R}$ when nothing is known about $\gamma$, (ii) $\mathcal{S}=\left[\gamma_{\min }, \gamma_{\max }\right]$ when it is easy to specify SNR bounds within which the observation lies with a high probability, or (iii) $\mathcal{S}=\left\{\gamma_{0}\right\}$ when (asymptotically) optimal properties are required for a specific SNR $\gamma_{0}$ and not necessarily for other values. Other approaches, such as marginalization, are possible when prior information are available on the SNR distribution. 


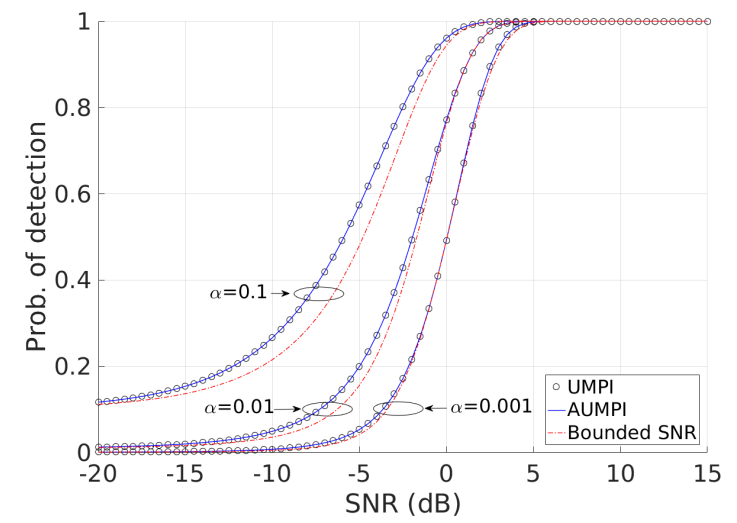

Fig. 1. Probability of detection versus SNR $\gamma, n=5, N=50, \tau=0.25$, $\rho_{0}=\tau, \rho_{1}=0.75, \mathcal{S}=[\gamma-3, \gamma+3] \mathrm{dB}$.

\section{NUMERICAL RESULTS}

Simulation examples illustrating the analytic results found in Sec. IV are provided. Three tests are compared : the AUMPI test $\mathcal{T}_{\alpha}$, the test $\mathcal{T}_{\alpha}^{\prime}$ with bounded SNR where $\mathcal{S}=\left[\gamma_{\min }, \gamma_{\max }\right]$, and the UMPI test that compares the likelihood ratio (14) to a threshold such that the size $\alpha$ is satisfied. This UMPI test does not exist when $\rho_{0}$ and $\rho_{1}$ are not provided and is therefore used as a performance bound. For all the simulations, $n=5$ and the tolerance is set to $\tau=0.25$. The percentage of the energy of $\boldsymbol{z}$ in the subspace $\langle\mathbf{H}\rangle$ under hypothesis $\mathcal{H}_{1}$ is set to $\rho_{1}=0.75$. For the UMPI bound, $\rho_{0}$ is set to $\rho_{0}=\tau$.

Fig. 1 shows the probability of detection versus SNR $\gamma$ for three different values of level $\alpha$, with $N=50, \mathcal{S}=[\gamma-3, \gamma+$ $3] \mathrm{dB}$. It can be seen that the performance gap between the AUMPI test $\mathcal{T}_{\alpha}$ and the UMPI bound is insignificant for this example. The loss due to unknown SNR is mostly visible for low SNR signals and for a high value of $\alpha$. Such a loss is explained by the conservative behavior of the test $\mathcal{T}_{\alpha}^{\prime}$.

Fig. 2 illustrates the performance loss induced by a lack of knowledge on the true SNR value. The probability of detection is shown as a function of the SNR gap $\Delta_{\gamma}$, defined in $\mathrm{dB}$ as $\Delta_{\gamma}=\gamma-\gamma_{\min }=\gamma_{\max }-\gamma$. As expected, the performance deteriorates with increasing uncertainty on the SNR. This is mostly detrimental for high sizes $\alpha$. It can be noticed that for a gap $\Delta_{\gamma}$ greater than $6 \mathrm{~dB}$, there is no additional loss due to additional SNR uncertainty. Such a figure can be relevant for engineers to know how much effort is needed to design an efficient SNR estimator, possibly used before detection.

\section{CONCLUSIONS}

The proposed asymptotic formulation of Karlin Rubin's theorem states that, for composite hypothesis testing, the comparison of a scalar statistic to a threshold leads to an AUMP test if this statistic converge in distribution to a random variable having a monotone increasing likelihood ratio. This result has then been applied to derive an AUMP invariant test to a subspace cone detection problem where the UMP (invariant) test does not exist. If we are given SNR, the numerical results indicate that the AUMPI test approaches the UMPI performance bound. For unknown SNR, a robust test

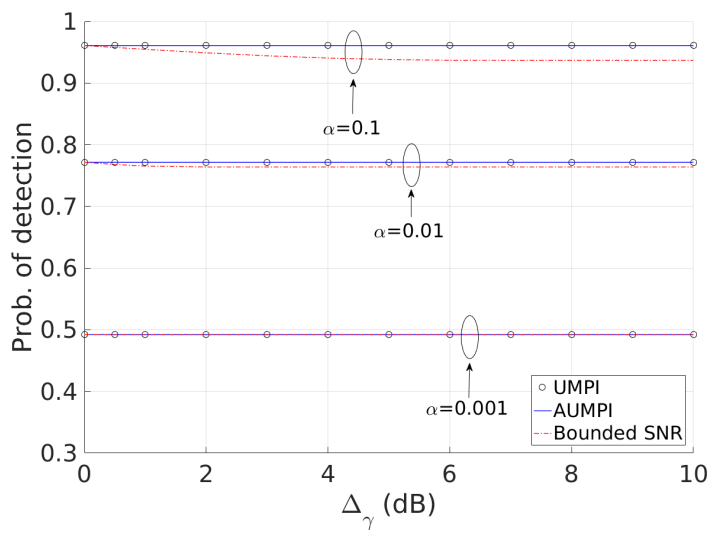

Fig. 2. Probability of detection versus the SNR gap, $n=5, N=50$, $\tau=0.25, \rho_{0}=\tau, \rho_{1}=0.75, \gamma=0 \mathrm{~dB}$.

derived from the AUMPI test is proposed and shown to offer a limited performance loss compared to the UMPI bound.

\section{APPENDiX A}

PROOF OF PROP. 1

The specificity of this asymptotic version of NeymanPearson's lemma is handled thanks to the Portmanteau theorem [13, Th. 2.1]. More specifically, according to this theorem we have

$$
\forall \mathcal{T} \in \xi_{\boldsymbol{Y}} \quad \lim _{n} \mathbb{E}_{\theta}\left[\mathcal{T}\left(\boldsymbol{Y}_{n}\right)\right]=\mathbb{E}_{\theta}[\mathcal{T}(\boldsymbol{Y})] .
$$

Define $\tilde{\varphi} \in \xi_{\boldsymbol{Y}}$ such that $\mathbb{E}_{\theta_{0}}[\tilde{\varphi}(\boldsymbol{Y})] \leq \alpha$. By using the definitions of $\varphi$ and $\tilde{\varphi}$, we have the following inequality for all $\boldsymbol{y}$ in $\mathbb{R}^{N}$

$$
(\tilde{\varphi}(\boldsymbol{y})-\varphi(\boldsymbol{y}))\left(f_{\boldsymbol{Y}}^{\theta_{1}}(\boldsymbol{y})-\eta f_{\boldsymbol{Y}}^{\theta_{0}}(\boldsymbol{y})\right) \leq 0
$$

yielding

$$
\mathbb{E}_{\theta_{1}}[\tilde{\varphi}(\boldsymbol{Y})]-\mathbb{E}_{\theta_{1}}[\varphi(\boldsymbol{Y})] \leq \eta\left(\mathbb{E}_{\theta_{0}}[\tilde{\varphi}(\boldsymbol{Y})]-\mathbb{E}_{\theta_{0}}[\varphi(\boldsymbol{Y})]\right)
$$

As the right term of the previous inequality is negative and by applying the Portmanteau theorem, we can deduce that

$$
\begin{aligned}
& \mathbb{E}_{\theta_{1}}[\tilde{\varphi}(\boldsymbol{Y})]-\mathbb{E}_{\theta_{1}}[\varphi(\boldsymbol{Y})] \\
& \quad=\lim _{n}\left\{\mathbb{E}_{\theta_{1}}\left[\tilde{\varphi}\left(\boldsymbol{Y}_{n}\right)\right]-\mathbb{E}_{\theta_{1}}\left[\varphi\left(\boldsymbol{Y}_{n}\right)\right]\right\} \leq 0 .
\end{aligned}
$$

Therefore the test $\varphi$ is AUMP among all tests in $\xi_{\boldsymbol{Y}}$.

To show that $\varphi$ is unbiased, we note that $\forall \boldsymbol{y} \in \mathbb{R}^{N}$ and $\forall \gamma \in[0,1]$

$$
\begin{aligned}
& (\gamma-\varphi(\boldsymbol{y}))\left(f_{\boldsymbol{Y}}^{\theta_{1}}(\boldsymbol{y})-\eta f_{\boldsymbol{Y}}^{\theta_{0}}(\boldsymbol{y})\right) \leq 0 \\
\Rightarrow & \gamma-\mathbb{E}_{\theta_{1}}[\varphi(\boldsymbol{Y})] \leq \eta(\gamma-\alpha) .
\end{aligned}
$$

If we set $\gamma=\alpha$, we then get $\mathbb{E}_{\theta_{1}}[\varphi(\boldsymbol{Y})] \geq \alpha$.

\section{APPENDIX B}

\section{PROOF OF PROP. 2}

The proof can be broken up into three major steps. The first step is to demonstrate that the test $\varphi^{\prime}$ is AUMP for the detection problem (5) of Prop. 1. The second is to prove that $\varphi^{\prime}$ is also AUMP when the alternative hypothesis $\mathcal{H}_{1}$ is composite, and the last step is to obtain the same result when 
the null hypothesis $\mathcal{H}_{0}$ is composite as well. Once Prop. 1 is established, the first two steps are similar to the proof of the non-asymptotic Karlin-Rubin theorem. Step 3 is more specific.

Lemma 1. For the detection problem (5), if the likelihood ratio $\Lambda_{\theta_{0}, \theta_{1}}(\boldsymbol{y})$ is a strictly increasing function of $V(\boldsymbol{y})$, then the test $\varphi^{\prime}$ is AUMP among all the tests belonging to $\xi_{\boldsymbol{Y}}$.

Proof. From definition (4), the AUMP test for problem (5) can be restated as

$$
\varphi(\boldsymbol{y})= \begin{cases}1 & \text { if } \Lambda_{\theta_{0}, \theta_{1}}(\boldsymbol{y}) \geq \eta \\ 0 & \text { otherwise }\end{cases}
$$

If we set $\Lambda_{\theta_{0}, \theta_{1}}(\boldsymbol{y})=h_{\theta_{0}, \theta_{1}}(V(\boldsymbol{y}))$, then

$$
\varphi(\boldsymbol{y})= \begin{cases}1 & \text { if } h_{\theta_{0}, \theta_{1}}(V(\boldsymbol{y})) \geq \eta \\ 0 & \text { otherwise. }\end{cases}
$$

Therefore, if $h_{\theta_{0}, \theta_{1}}(V(\boldsymbol{y}))$ is strictly increasing in $V(\boldsymbol{y})$ then $\varphi^{\prime}$ is AUMP because $h_{\theta_{0}, \theta_{1}}$ admits an inverse function so that

$$
\varphi(\boldsymbol{y})=\varphi^{\prime}(\boldsymbol{y})= \begin{cases}1 & \text { if } V(\boldsymbol{y}) \geq \lambda \\ 0 & \text { otherwise }\end{cases}
$$

where $\lambda$ is defined according to (8) and satisfies $\lambda=h_{\theta_{0}, \theta_{1}}^{-1}(\eta)$.

Lemma 2. The test $\varphi^{\prime}$ is AUMP among all the tests belonging to $\xi_{Y}$ for testing

$$
\left\{\begin{array}{l}
\mathcal{H}_{0}^{\prime \prime}: \theta=\theta_{0} \\
\mathcal{H}_{1}^{\prime \prime}: \theta>\theta_{0}
\end{array}\right.
$$

Proof. From (7) and (8), it can be noticed that as opposed to $\varphi$, the test $\varphi^{\prime}$ does not depend on $\theta_{1}$. It only depends on the two parameters $\theta_{0}$ and $\alpha$. Moreover $h_{\theta_{0}, \theta_{1}}$ is strictly increasing in $V$ for any $\theta_{0}<\theta_{1}$. Consequently, the test is AUMP among the tests belonging to $\xi_{\boldsymbol{Y}}$ for any $\theta_{1}$ and $\theta_{0}$ satisfying $\theta_{0}<\theta_{1}$.

For the third and last step of the proof, we must show that $\varphi^{\prime}$ is also AUMP when $\mathcal{H}_{0}$ is composite. Consequently, we must prove that

$$
\lim _{n} \sup _{\theta \leq \theta_{0}} \mathbb{E}_{\theta}\left[\varphi^{\prime}\left(\boldsymbol{Y}_{n}\right)\right] \leq \mathbb{E}_{\theta_{0}}\left[\varphi^{\prime}(\boldsymbol{Y})\right]=\alpha .
$$

We proceed in two stages.

Lemma 3. The test $\varphi^{\prime}$ satisfies

$$
\lim _{n} \sup _{\theta \leq \theta_{0}} \mathbb{E}_{\theta}\left[\varphi^{\prime}\left(\boldsymbol{Y}_{n}\right)\right]=\sup _{\theta \leq \theta_{0}} \mathbb{E}_{\theta}\left[\varphi^{\prime}(\boldsymbol{Y})\right]
$$

Proof. According to the Portmanteau theorem, we have: $\lim _{n} \mathbb{E}_{\theta}\left[\varphi^{\prime}\left(\boldsymbol{Y}_{n}\right)\right]=\mathbb{E}_{\theta}\left[\varphi^{\prime}(\boldsymbol{Y})\right]$. Therefore, $\forall \boldsymbol{y} \in \mathbb{R}^{N}, \forall \epsilon>$ $0, \exists N_{\epsilon, \boldsymbol{y}} \in \mathbb{N}, \forall n \geq N_{\epsilon, \boldsymbol{y}}$

$$
-\epsilon \leq \int_{\mathbb{R}^{N}} \varphi^{\prime}(\boldsymbol{y}) f_{\boldsymbol{Y}_{n}}^{\theta}(\boldsymbol{y}) d \boldsymbol{y}-\int_{\mathbb{R}^{N}} \varphi^{\prime}(\boldsymbol{y}) f_{\boldsymbol{Y}}^{\theta}(\boldsymbol{y}) d \boldsymbol{y} \leq \epsilon,
$$

yielding,

$$
\mathbb{E}_{\theta}\left[\varphi^{\prime}(\boldsymbol{Y})\right]-\epsilon \leq \mathbb{E}_{\theta}\left[\varphi^{\prime}\left(\boldsymbol{Y}_{n}\right)\right] \leq \mathbb{E}_{\theta}\left[\varphi^{\prime}(\boldsymbol{Y})\right]+\epsilon .
$$

Consequently, $\forall \theta \leq \theta_{0}$,

$$
\left\{\begin{array}{l}
\mathbb{E}_{\theta}\left[\varphi^{\prime}\left(\boldsymbol{Y}_{n}\right)\right] \leq \sup _{\theta \leq \theta_{0}} \mathbb{E}_{\theta}\left[\varphi^{\prime}(\boldsymbol{Y})\right]+\epsilon \\
\mathbb{E}_{\theta}\left[\varphi^{\prime}(\boldsymbol{Y})\right] \leq \sup _{\theta \leq \theta_{0}} \mathbb{E}_{\theta}\left[\varphi^{\prime}\left(\boldsymbol{Y}_{n}\right)\right]+\epsilon
\end{array}\right.
$$

$$
\Rightarrow \begin{cases}\sup _{\theta \leq \theta_{0}} \mathbb{E}_{\theta}\left[\varphi^{\prime}\left(\boldsymbol{Y}_{n}\right)\right] \leq \sup _{\theta \leq \theta_{0}} \mathbb{E}_{\theta}\left[\varphi^{\prime}(\boldsymbol{Y})\right]+\epsilon \\ \sup _{\theta \leq \theta_{0}} \mathbb{E}_{\theta}\left[\varphi^{\prime}(\boldsymbol{Y})\right] \leq \sup _{\theta \leq \theta_{0}} \mathbb{E}_{\theta}\left[\varphi^{\prime}\left(\boldsymbol{Y}_{n}\right)\right]+\epsilon\end{cases}
$$

From the previous equation, we deduce that $\forall \theta \leq \theta_{0}$

$$
\left|\sup _{\theta \leq \theta_{0}} \mathbb{E}_{\theta}\left[\varphi^{\prime}\left(\boldsymbol{Y}_{n}\right)\right]-\sup _{\theta \leq \theta_{0}} \mathbb{E}_{\theta_{0}}\left[\varphi^{\prime}(\boldsymbol{Y})\right]\right| \leq \epsilon,
$$

which concludes the proof.

Lemma 4. The test $\varphi^{\prime}$ satisfies $\sup _{\theta \leq \theta_{0}} \mathbb{E}_{\theta}\left[\varphi^{\prime}(\boldsymbol{Y})\right]=$ $\mathbb{E}_{\theta_{0}}\left[\varphi^{\prime}(\boldsymbol{Y})\right]$.

Proof. We consider the test $\varphi^{\prime}$ used for testing $\left\{\begin{array}{l}\mathcal{H}_{0}^{\prime \prime}: \theta=\theta_{2} \\ \mathcal{H}_{1}^{\prime \prime}: \theta>\theta_{2}\end{array}, \quad\right.$ with $\theta_{2}<\theta_{0}$. Since $\varphi^{\prime}=\varphi$ and the test $\varphi$ is unbiased, we can deduce that $\mathbb{E}_{\theta_{2}}\left[\varphi^{\prime}(\boldsymbol{Y})\right] \leq \mathbb{E}_{\theta_{0}}\left[\varphi^{\prime}(\boldsymbol{Y})\right], \quad \forall \theta_{2}<\theta_{0} . \quad$ Hence $\sup _{\theta \leq \theta_{0}} \mathbb{E}_{\theta}\left[\varphi^{\prime}(\boldsymbol{Y})\right]=\mathbb{E}_{\theta_{0}}\left[\varphi^{\prime}(\boldsymbol{Y})\right]$

From Lemma (3) and (4), we can conclude that (25) is satisfied. The proof of Theorem 2 is then obtained by combining Lemma 1, 2 and the inequality (25).

\section{REFERENCES}

[1] S. Karlin and H. Rubin, "Distributions possessing a monotone likelihood ratio," J. Amer. Statist. Assoc., vol. 51, pp. 637 - 643, Feb. 1956.

[2] E. L. Lehmann and J. P. Romano, Testing statistical hypotheses, Springer, 3rd edition, 2005

[3] L. L. Scharf, Statistical Signal Processing: Detection, Estimation, and Time Series Analysis, Addison-Wesley, 1991.

[4] R. T. Behrens and L. L. Scharf, "Signal processing applications of oblique projection operators," IEEE Transactions on Signal Processing, vol. 42, no. 6, pp. 1413-1424, 1994.

[5] T. T. Liu, L. R. Frank, E. C. Wong, and R. B. Buxton, "Detection power, estimation efficiency, and predictability in event-related fmri," Neuroimage, vol. 13, no. 4, pp. 759-773, 2001.

[6] D. Manolakis, D. Marden, and G. A. Shaw, "Hyperspectral image processing for automatic target detection applications," Lincoln laboratory journal, vol. 14, no. 1, pp. 79-116, 2003.

[7] L. L. Scharf and B. Friedlander, "Matched Subspace Detectors," IEEE Trans. Signal Process., vol. 42, no. 8, pp. 2146 - 2157, 1994.

[8] S. Ramprashad, T. W Parks, and R. Shenoy, "Signal modeling and detection using cone classes," IEEE transactions on signal processing, vol. 44, no. 2, pp. 329-338, 1996.

[9] O. Besson, "Detection of a signal in linear subspace with bounded mismatch," IEEE Transactions on Aerospace and Electronic Systems, vol. 42 , no. 3, 2006.

[10] F.-X. Socheleau and D. Pastor, "Testing the energy of random signals in a known subspace: An optimal invariant approach," IEEE Signal Processing Letters, vol. 21, no. 10, pp. 1182-1186, 2014.

[11] M. N. Desai and R. S. Mangoubi, "Robust gaussian and non-gaussian matched subspace detection," IEEE Transactions on Signal Processing, vol. 51, no. 12, pp. 3115-3127, 2003.

[12] F.-X. Socheleau, E. Leroy, A. Carvallo Pecci, F. Samaran, J. Bonnel, and J.-Y. Royer, "Automated detection of antarctic blue whale calls," $J$. Acoust. Soc. Am., vol. 138, no. 5, pp. 3105-3117, 2015.

[13] P. Billingsley, Probability and Measure, 3rd edition, Wiley, 1995.

[14] F.-X. Socheleau and F. Samaran, "Detection of Mysticete Calls: a Sparse Representation-Based Approach, Research report RR-2017-04-SC," Oct. 2017, https://hal.archives-ouvertes.fr/hal-01736178/document.

[15] N. L. Johnson, S. Kotz, and N Balakrishnan, Continous Univariate distributions, 2nd edition, vol. 2, John Wiley \& Sons, Inc, 1995.

[16] M. L. Eaton, Multivariate statistics. A vector space approach, Wiley, 1983. 